\title{
Patientennutzen und -wünsche verstehen
}

\author{
Eine Befragung zeigte: Patienten erwarten von ihrem Hausarzt vor allem Fachwis- \\ sen, Kommunikationskompetenz und Freundlichkeit. Eine grundlegende Rolle spielt \\ der Aufbau von Vertrauen.
}

Carolin Dengler-Voss ${ }^{a}$, Rödiger Voss ${ }^{b}$

a Dr. med., Fachärztin für Allgemeine Innere Medizin

b Prof. Dr., Professor für Betriebswirtschaftslehre und Lernmanagement FH Zürich

\section{Literatur}

1 Dengler-Voss C, Voss R. Welche Ansprüche haben meine Patienten an mich und welche Wünsche stecken dahinter. PrimaryCare. 2014;14(18):289-91.

2 Diviani-Preiswerk S. Wie sag ich's meinen Patienten? Schweiz Ärztezeitung. 2014; 95(15):616-8.

Korrespondenz:

Dr. med. Carolin Dengler-Voss Institut für Rechtsmedizin Verkehrsmedizin und Forensische Psychiatrie

Kurvenstrasse 31

CH-8006 Zürich

carolin.dengler-voss[at] irm.uzh.ch
Der Fall: Er fühle sich durch das neue Blutdruckmedikament wie «am Tüfel ab em Charre gheit», bekundete der Patient Herr K. anlässlich der Kontrollkonsultation bei seinem Hausarzt Dr. V. Das sei kaum möglich, betont Dr. V., es handle sich derzeit noch um eine «homöopathische» Dosierung. Herr K. fühlte sich von seinem Hausarzt nicht ernst genommen. Er hätte sich in dieser Situation von ihm mehr Empathie erwartet. Infolge seiner Enttäuschung suchte er diesen Arzt danach nicht wieder auf.

In der Praxis sind viele vergleichbare Beispiele denkbar, in denen es infolge unterschiedlicher Erwartungen, Meinungen und Einstellungen von Patient und Hausarzt $\mathrm{zu}$ missverständlicher Kommunikation und im schlimmsten Fall zu einem Abbruch der Patienten-Arzt-Beziehung kommen kann. Aus diesem Problemkreis heraus entstand die Idee einer Studie mit dem Ziel, die Ansprüche von Patienten an ihren Hausarzt genauer zu untersuchen [1]. Zu diesem Zweck wurden in der Deutschschweiz 35 qualitative Tiefen-Interviews geführt, um die Erwartungen an einen Hausarzt, den damit verbundenen positiven Nutzen und die dahinterliegenden Werte zu ermitteln.

Die gewonnenen Daten wurden softwareunterstützt einer inhaltlichen Analyse unterzogen. Hierbei wurde ersichtlich, dass Patienten von Hausärzten sieben wesentliche Kompetenzen und Qualifikationen wünschen: Fachwissen, Kommunikationskompetenz, Freundlichkeit, Empathie, Proaktivsein, Berufserfahrungen und Geduld. Besonders relevant waren davon Fachwissen, Kommunikationskompetenz und Freundlichkeit. Zudem liessen sich auf Patientenseite verschiedene Nutzeneigenschaften ableiten, die mit den genannten hausärztlichen Kompetenzen in Verbindung gebracht wurden. Im Vordergrund standen hier das Vertrauen, die Stellung einer korrekten Diagnose und die Heilung. Wichtig war den befragten Personen auch ein respektvoller Umgang, das Gefühl, ernst genommen zu werden, mitreden zu dürfen und eine effiziente Behandlung zu erfahren. Im Altersvergleich der Exploranden liessen sich zum Teil Unterschiede darstellen: Den jüngeren befragten Personen war es beispielsweise wichtig, dass die Behandlung zeiteffizient und reibungslos abläuft und sie so rasch wie möglich wieder arbeitsfähig werden. Auch legten sie ein grösseres Augenmerk auf die Internetpräsenz ihrer Ärzte sowie auf deren dokumentierten Lebenslauf. Als übergeordneter Wert stellte sich in unserem Kollektiv erwartungsgemäss das (Wieder-)Erlangen der Gesundheit dar. Im gesunden Zustand können Individuen nach Freude, Genuss und Lust (Hedonismus) streben. Wichtige Werte waren für die Befragten jedoch auch das Gefühl des Wohlbefindens und der Sicherheit.

Hervorzuheben ist die zentrale Rolle des Vertrauens. Wenn die Patienten Vertrauen entwickeln, können sie sich öffnen und frei mitteilen, auch wenn es sich um sehr intime Gegebenheiten handelt (Abb. 1). Alle genannten hausärztlichen Kompetenzen zeigen eine direkte oder indirekte Verbindung zum Vertrauen. Besonders wichtig für die Vertrauensbildung waren Kommunikationskompetenz, Empathie und Proaktivsein, worunter das aktive Nachfragen des Arztes sowie sein gezeigter Einsatz zusammenzufassen sind. Erst wenn Patienten dem Arzt vertrauen und sich ihm öffnen können, entwickeln sie ein Gefühl des Wohlfühlens und der Sicherheit. Zusammenfassend veranschaulichen die Studienergebnisse unter anderem, dass insbesondere zwischenmenschliche Faktoren für die Vertrauensbildung und damit für den Kontakt zwischen Patient und Hausarzt sehr wichtig sind, was nicht zuletzt zu zufriedenen Patienten führt, die ihren Hausarzt in Problemlagen wieder aufsuchen. Ärzte können diese Fähigkeiten durch Rollenspiele und weitere Übungsmethoden mit erfahrenen Kollegen, Kommunikationstrainern oder speziellen Weiterbildungsangeboten [2] optimieren. 